\title{
The Ionic Mechanisms of Triggered Atrial Activity Under a TBX5-driven Regulatory Network
}

\author{
Andy C Y Lo ${ }^{1}$, Jieyun Bai $^{2}$, Patrick A Gladding ${ }^{3}$, Jichao Zhao ${ }^{1}$ \\ ${ }^{1}$ Auckland Bioengineering Institute, University of Auckland, Auckland, New Zealand \\ ${ }^{2}$ Department of Electronic Engineering, Jinan University, Guangzhou, China \\ ${ }^{3}$ Department of Cardiology, Waitemata District Health Board, Auckland, New Zealand
}

\begin{abstract}
Atrial fibrillation $(A F)$ is the most prevalent arrhythmia in clinical practice, yet the pathophysiology by which genetic factors can increase the risk of $A F$ is not well understood. Recently, a multitiered transcriptional network, driven by a T-box transcription factor gene TBX5 and a paired-like homeodomain transcription factor 2 gene PITX2 was discovered. This transcriptional network regulates gene expressions associated with ion channels in a complex fashion, and through mice knockout studies, it was found that reducing the expression of TBX5 altered the gene expressions of certain types of ion channels and generated abnormal depolarizations in the form of early afterdepolarizations, delayed afterdepolarizations, or spontaneous triggered action potentials. To systematically investigate the ionic mechanisms by which impaired TBX5 can lead to $A F$, we integrated the calcium dynamics of the Grandi et al. model into the Courtemanche-Ramirez-Nattel model. Our model reproduced all forms of abnormal depolarizations observed in TBX5 knockout atrial myocytes. Furthermore, we discovered that the remodeling of the inward-rectifier potassium channel $\left(I_{K I}\right)$ and the $L$ type calcium channel $\left(I_{C a L}\right)$, due to impaired TBX5, causes an elevation in the concentration of calcium $\left(\left[\mathrm{Ca}^{2+}\right]\right)$, which reactivates the sodium-calcium exchanger $\left(I_{\mathrm{NaCa}}\right)$ and $I_{C a L}$ to generate abnormal depolarizations.
\end{abstract}

\section{Introduction}

Atrial fibrillation (AF) is the most commonly encountered cardiac arrhythmia in clinical practice and is associated with an increased risk of heart failure, stroke, and mortality [1]. Antiarrhythmic drug (AAD) treatment by targeting specific ionic channels is the first-line therapy for patients with AF. However, due to our limited understanding of the precise ionic mechanisms within the human heart, AAD treatment has limited efficacy and is even prone to adverse effects [2].

Within the past decade, there has been a plethora of evidence based on studies from referral populations and in the community to suggest that $\mathrm{AF}$ is heritable, whereby having a family member with AF leads to a $40 \%$ increased risk of developing the arrhythmia [3]. To identify the genes responsible for the heritability of AF, several largescale genome-wide association studies (GWAS) were conducted and had implicated the T-box transcription factor gene TBX5 and the paired-like homeodomain transcription factor 2 gene PITX 2 to be strongly associated with $\mathrm{AF}$ [4]. Both transcription factors were primarily known for the roles they play in cardiac development during embryogenesis.

Through mice knockout studies, Nadadur et al. [5] demonstrated that impairment of TBX5 resulted in diminished gene expressions of ionic and calcium handling channels, leading to action potential duration (APD) prolongation and abnormal depolarizations, i.e., early and delayed afterdepolarizations (EADs and DADs), and spontaneous triggered action potentials (STAPs), which are known substrates to AF [6]. In contrast, impairment of PITX2 instead resulted in the upregulation of these gene expressions and the shortening of APD. Furthermore, knockout of PITX2 in the TBX5 deficient mice was able to rescue reduced ionic and calcium handling channel gene expressions and abolish the presence of abnormal depolarizations, suggesting that both TBX5 and PITX2 work antagonistically with one another in regulating the aforementioned channel gene expressions through a TBX5-driven regulatory network.

Because of these complex interactions, it is imperative that we utilize a computational model as it provides a powerful framework for investigating the electrical properties of atrial myocytes under impaired TBX5 through its ability to dissect highly intertwined contributing factors and to simulate control studies. Hence, to elucidate the exact mechanisms by which impaired TBX5 in the regulatory network can lead to the onset of abnormal depolarizations and to identify potential AAD targets, we developed a biophysics based model of the atrial myocyte that incorporates these channel gene expression changes due to impaired TBX5 and is capable of reproducing EADs and STAPs/DADs.

\section{Method}

We developed a new human atrial cellular model (CRNGrandi) by integrating the calcium dynamics of the Grandi et al. model [7] into the Courtemanche-Ramirez- Nattel 
model [8] to reproduce abnormal depolarizations (Fig 1). Alterations to the ionic and $\mathrm{Ca}^{2+}$ handling channels due to impaired TBX5 were made in the CRN-Grandi model as listed in Table 1. These changes were based primarily on the proposed changes as stated by Bai et al. [9] for homogeneous TBX5 knockout.

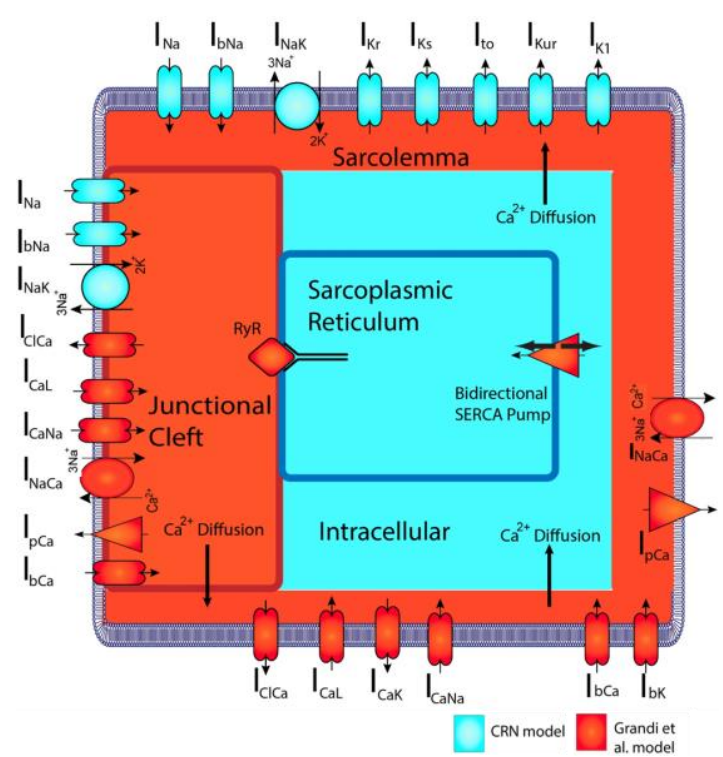

Figure 1: Schematic representation of currents, pumps, exchangers, and compartments in the CRN-Grandi model.

In each simulation run, starting with initial conditions taken from the Grandi et al. model, a train of stimuli with a duration of $5 \mathrm{~ms}$ was applied at a frequency of $1 \mathrm{~Hz}$ up till $30.1 \mathrm{sec}$ into the simulation. In our subsequent figures, we indicate the timings when a stimulus is applied with $\boldsymbol{\nabla}$.

To investigate the impact the remodeling of each channel has on the action potential (AP), we first ran a series of simulations where we separately implemented the remodeling of each current. From there, we then ran simulations first with the remodeling of $\mathrm{I}_{\mathrm{K} 1}$ implemented, then with the remodeling of $\mathrm{I}_{\mathrm{Kur}}$, and so forth one at a time until all the remodeling of all affected channels were implemented into the model.

A similar study was then conducted on the main channels responsible for EAD and STAP/DAD generation to probe how the remodeling of each of those currents affects the concentration of calcium in both the junctional cleft $\left(\left[\mathrm{Ca}^{2+}\right]_{\mathrm{j}}\right)$ and sarcolemma $\left(\left[\mathrm{Ca}^{2+}\right]_{\mathrm{sl}}\right)$ during the systolic and diastolic phase of an AP. The conductance changes for this study were chosen as the maximum changes possible without eliciting EADs and STAPs/DADs which would otherwise result in a drastic qualitative change in $\left[\mathrm{Ca}^{2+}\right]$ and making comparisons between the cases unfeasible.

Following this, we then performed a series of tests where we experimented with combinations of $\left[\mathrm{Ca}^{2+}\right]$ clamp up and $\left[\mathrm{Na}^{+}\right]$clamp down. Lastly, we replicated the inhibitory effects of AADs on $\mathrm{I}_{\mathrm{NaCa}}$ and $\mathrm{I}_{\mathrm{CaL}}$ by performing simulations with each one separately reduced to various levels.

\begin{tabular}{|c|c|c|c|c|c|c|c|}
\hline Current & $\mathrm{I}_{\mathrm{Na}}$ & $\mathrm{I}_{\text {to }}$ & $\mathrm{I}_{\mathrm{K} 1}$ & $\mathrm{I}_{\text {kur }}$ & $\mathrm{I}_{\text {CaL }}$ & SERCA & RyR \\
\hline $\begin{array}{c}\text { Conductance } \\
\text { Change }\end{array}$ & $-70 \%$ & $-65 \%$ & $-80 \%$ & $-58 \%$ & $+70 \%$ & $-58 \%$ & $-78 \%$ \\
\hline
\end{tabular}

\section{Results}

\section{The impact of ionic channel remodeling due to impaired TBX5}
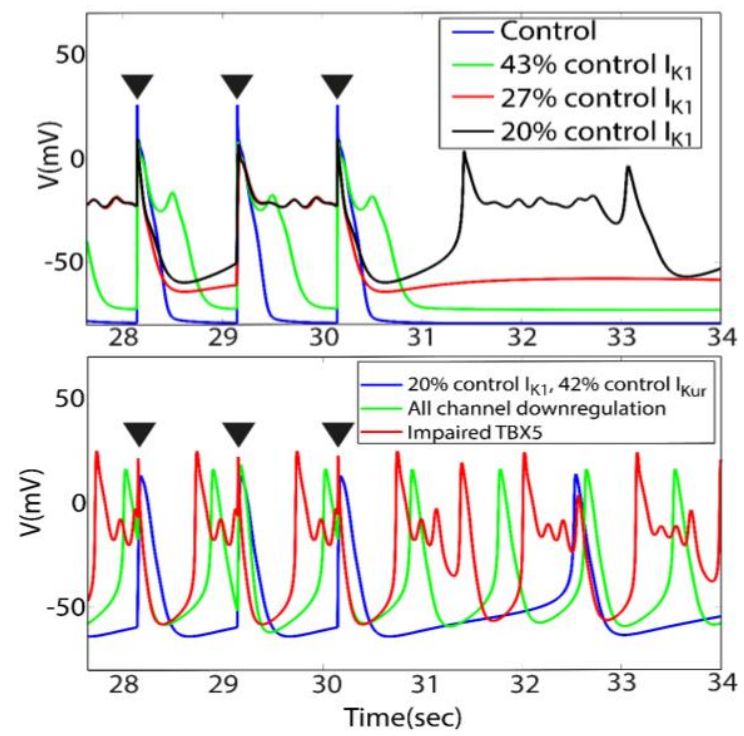

Figure 2: Changes to the AP due to variations in $\mathrm{I}_{\mathrm{K} 1}$ (up) and as more remodeled channels under impaired TBX5 were included (down).

From our initial series of simulations where we separately implemented the remodeling of each current, we identified $I_{K 1}$ as the most influential current. In the top panel of Figure 2, we show that variations in its reduction can generate different abnormal depolarizations. When $\mathrm{I}_{\mathrm{K} 1}$ was reduced to $43 \%$ of its control value, the model was able to produce EADs (green), while at $27 \%$ of its control value, EADs and a DAD occurred instead (red). At $20 \%$ of its control value however, the model was able to generate not only EADs, but also STAPs (black).

EADs were abolished when we included the downregulation of $\mathrm{I}_{\mathrm{Kur}}$ alongside $20 \% \mathrm{I}_{\mathrm{K} 1}$ (Fig 2 down, blue). Including the remodeling of the remaining ionic channels and the $\mathrm{Ca}^{2+}$ handling channels impacted by TBX5 knockout except $\mathrm{I}_{\mathrm{CaL}}$ only increased the frequency of the STAPs (Fig 2 down, green). Lastly, including the 
upregulation of $\mathrm{I}_{\mathrm{CaL}}$ was able to counteract $\mathrm{I}_{\mathrm{Kur}}$, as EADs were present in the simulation once more (Fig 2 down, red).

\section{Remodeled systolic and diastolic $\left[\mathrm{Ca}^{2+}\right]_{\mathrm{j}}$ and $\left[\mathrm{Ca}^{2+}\right]_{\mathrm{sl}}$}

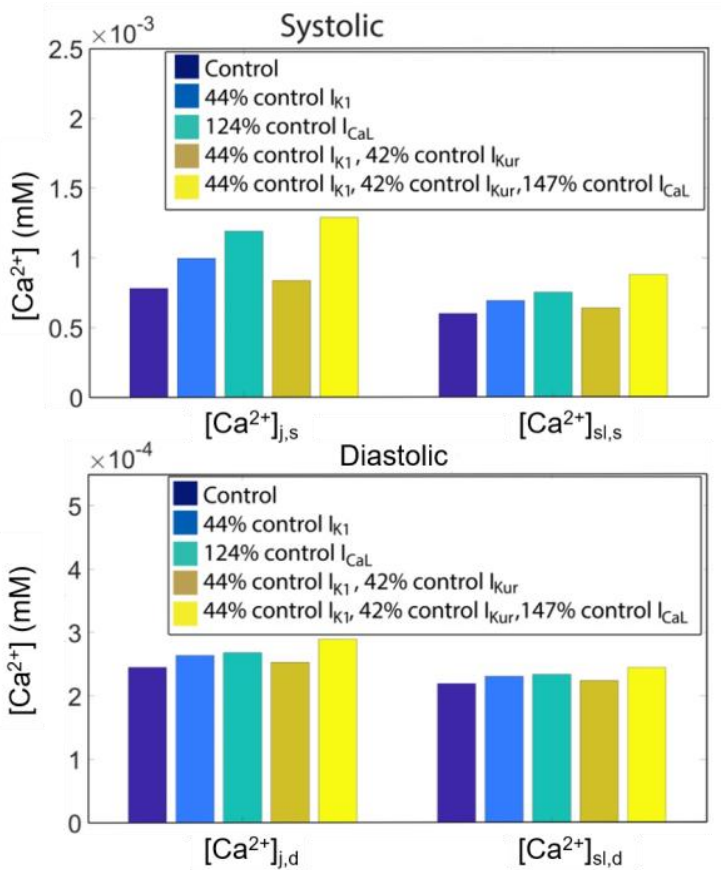

Figure 3: Comparing systolic and diastolic $\left[\mathrm{Ca}^{2+}\right]_{\mathrm{j}}$ and $\left[\mathrm{Ca}^{2+}\right]_{\mathrm{sl}}$ at the last stimulated AP between the control and the three remodeling cases.

Having identified $\mathrm{I}_{\mathrm{K} 1}, \mathrm{I}_{\mathrm{Kur}}$, and $\mathrm{I}_{\mathrm{CaL}}$ to be the main channels responsible for EAD and STAP/DAD generation, we then conducted a separate control study on these channels exclusively to see how remodeling of these channels affected systolic and diastolic $\left[\mathrm{Ca}^{2+}\right]_{\mathrm{j}}$ and $\left[\mathrm{Ca}^{2+}\right]_{\mathrm{sl}}$. With $44 \% \mathrm{I}_{\mathrm{K} 1}$ or $124 \% \mathrm{I}_{\mathrm{CaL}}$, both systolic and diastolic $\left[\mathrm{Ca}^{2+}\right]_{\mathrm{j}}$ and $\left[\mathrm{Ca}^{2+}\right]_{\mathrm{sl}}$ were increased (Fig 3, light blue and turquoise). Systolic $\left[\mathrm{Ca}^{2+}\right]_{\mathrm{j}}$ and $\left[\mathrm{Ca}^{2+}\right]_{\mathrm{sl}}$ decreased when $42 \% \mathrm{I}_{\mathrm{Kur}}$ was incorporated alongside $44 \% \mathrm{I}_{\mathrm{K} 1}$, but diastolic $\left[\mathrm{Ca}^{2+}\right]_{\mathrm{j}}$ and $\left[\mathrm{Ca}^{2+}\right]_{\mathrm{sl}}$ only decreased slightly (Fig 3, dark yellow). When $147 \% \mathrm{I}_{\mathrm{CaL}}$ was incorporated alongside $44 \%$ $\mathrm{I}_{\mathrm{K} 1}$ and $42 \% \mathrm{I}_{\mathrm{Kur}}$, both systolic and diastolic $\left[\mathrm{Ca}^{2+}\right]_{\mathrm{j}}$ and $\left[\mathrm{Ca}^{2+}\right]_{\mathrm{sl}}$ were reversed (Fig 3, light yellow).

Clamping down $\left[\mathrm{Ca}^{2+}\right]_{\mathrm{j}}$ and $\left[\mathrm{Ca}^{2+}\right]_{\mathrm{sl}}$, or clamping up $\left[\mathrm{Na}^{+}\right]_{\mathrm{j}}$ and $\left[\mathrm{Na}^{+}\right]_{\mathrm{sl}}$ abolishes EADs and STAPs/DADs

We then determined if the generation of EADs and STAPs/DADs due to the impaired expression of TBX5 was dependent on $\left[\mathrm{Ca}^{2+}\right]$. Under impaired TBX5 conditions, we clamped $\left[\mathrm{Ca}^{2+}\right]_{\mathrm{j}}$ and $\left[\mathrm{Ca}^{2+}\right]_{\mathrm{sl}}$ down to $0.0003 \mathrm{mM}$, which was able to suppress EADs, but not STAPs (Fig 4, blue).

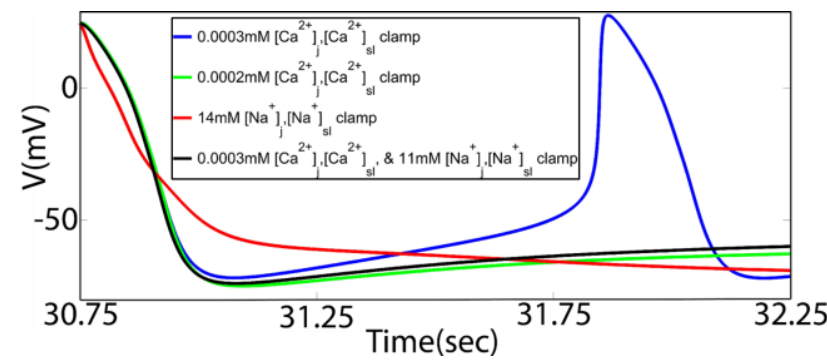

Figure 4: Changes to the AP under impaired TBX5 condition with different combinations of $\left[\mathrm{Ca}^{2+}\right]$ and $\left[\mathrm{Na}^{+}\right]$ clamps.

However, both were suppressed when the clamp was lowered to $0.0002 \mathrm{mM}$ (Fig 4, green). We also experimented with an $\left[\mathrm{Na}^{+}\right]$clamp, and found that both EADs and STAPs were also suppressed when $\left[\mathrm{Na}^{+}\right]_{j}$ and $\left[\mathrm{Na}^{+}\right]_{\mathrm{sl}}$ was clamped up to $14 \mathrm{mM}$ (Fig 4, red). Furthermore, if a $11 \mathrm{mM}$ clamp on $\left[\mathrm{Na}^{+}\right]_{\mathrm{j}}$ and $\left[\mathrm{Na}^{+}\right]_{\mathrm{sl}}$ was applied alongside a $0.0003 \mathrm{mM}$ clamp on $\left[\mathrm{Ca}^{2+}\right]_{\mathrm{j}}$ and $\left[\mathrm{Ca}^{2+}\right]_{\mathrm{sl}}$, then this too was able to suppress both EADs and STAPs (Fig 4, black).

Inhibiting $\mathbf{I}_{\mathrm{CaL}}$ or $\mathbf{I}_{\mathrm{NaCa}}$ abolishes EADs and STAPs/DADs

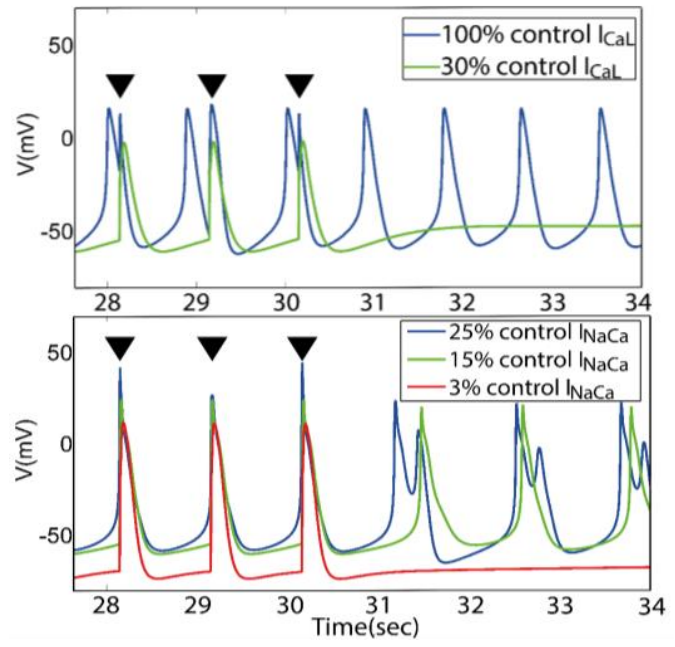

Figure 5: Changes to the AP under different levels of inhibition on $\mathrm{I}_{\mathrm{CaL}}$ (up) and $\mathrm{I}_{\text {Naca }}$ (down).

Because generation of impaired TBX5 induced EADs and STAPs/DADs was dependent on a relative high concentration of $\left[\mathrm{Ca}^{2+}\right]_{\mathrm{j}}$ and $\left[\mathrm{Ca}^{2+}\right]_{\mathrm{sl}}$, and a relative low concentration of $\left[\mathrm{Na}^{+}\right]_{\mathrm{j}}$ and $\left[\mathrm{Na}^{+}\right]_{\mathrm{sl}}$, we therefore hypothesized that $\mathrm{I}_{\mathrm{CaL}}$ and $\mathrm{I}_{\mathrm{NaCa}}$ are crucial currents that drive EADs and STAPs/DADs under such a condition.

When $\mathrm{I}_{\mathrm{CaL}}$ was returned to its control value, EADs were suppressed (Fig 5 up, blue). When $\mathrm{I}_{\mathrm{CaL}}$ was reduced to $30 \%$ of its control value, we found that this suppressed both EADs and STAPs instead (Fig 5 up, green). 
For $\mathrm{I}_{\mathrm{NaCa}}$, when we reduced it to $25 \%$ of its control value, we found that this suppressed EADs, but did not prevent STAPs and EADs on the STAPs (Fig 5 down, blue). EADs were completely suppressed with $\mathrm{I}_{\mathrm{NaCa}}$ reduced to $15 \%$ of its control value (Fig 5 down, green). Reduction of $\mathrm{I}_{\mathrm{NaCa}}$ down to only $3 \%$ of its control value was able to suppress both EADs and STAPs (Fig 5 down, red).

\section{Discussion}

It is generally accepted that STAPs/DADs are caused by an overload of $\mathrm{Ca}^{2+}$ in the sarcoplasmic reticulum (SR) during the diastolic phase of an AP, causing $\mathrm{Ca}^{2+}$ to be released and triggering the forward-model of the sodiumcalcium exchanger $\mathrm{I}_{\mathrm{NaCa}}$ to generate a transient inward current $[6,10,11]$. For EADs however, it is hypothesized that they can occur due to the prolongation of the APD, which in turns leads to the reactivation of $\mathrm{I}_{\mathrm{CaL}}$ and $\mathrm{I}_{\mathrm{Na}}[6$, $11,12]$ during phase 2 or 3 of an AP, or by the inward current from $\mathrm{I}_{\mathrm{NaCa}}$ due to elevated $\mathrm{Ca}^{2+}$ [11-13].

Under impaired TBX5, we found that the elevation in $\mathrm{Ca}^{2+}$ is instead caused by the remodeling of $\mathrm{I}_{\mathrm{K} 1}$ and $\mathrm{I}_{\mathrm{CaL}}$, which raises systolic and diastolic $\left[\mathrm{Ca}^{2+}\right]_{\mathrm{j}}$ and $\left[\mathrm{Ca}^{2+}\right]_{\mathrm{sl}}$. Sufficient elevation in systolic $\left[\mathrm{Ca}^{2+}\right]_{\mathrm{j}}$ and $\left[\mathrm{Ca}^{2+}\right]_{\mathrm{sl}}$ triggers $\mathrm{I}_{\mathrm{CaL}}$ and $\mathrm{I}_{\mathrm{NaCa}}$ to generate EADs during phase 2 or 3 of an $\mathrm{AP}$, while the same channels can also be triggered during the diastolic phase of an AP by a sufficient increase in diastolic $\left[\mathrm{Ca}^{2+}\right]_{\mathrm{j}}$ and $\left[\mathrm{Ca}^{2+}\right]_{\mathrm{sl}}$ to generate STAPs/DADs. Simulations where we inhibited $\mathrm{I}_{\mathrm{CaL}}$ and $\mathrm{I}_{\mathrm{NaCa}}$ separately demonstrate that both impaired TBX5-induced EADs and STAPs/DADs are dependent on both channels. Since a significantly higher inhibition on $\mathrm{I}_{\mathrm{NaCa}}$ is required to suppress both EADs and STAPs/DADs, we believe $\mathrm{I}_{\mathrm{NaCa}}$ to be the primary driver of these abnormal depolarizations, while $\mathrm{I}_{\mathrm{CaL}}$ plays more of a supporting role.

Our study expands upon the findings by Bai et al., where we have not only identified the remodeling of $\mathrm{I}_{\mathrm{K} 1}$ and $\mathrm{I}_{\mathrm{CaL}}$ to be the main initiators of impaired TBX5-induced EADS and STAPs/DADs, but we have also elucidated the mechanisms by which these abnormal depolarizations occur. Further investigation however is required to determine the precise mechanisms by which remodeling of $\mathrm{I}_{\mathrm{K} 1}$ and $\mathrm{I}_{\mathrm{CaL}}$ causes an elevation in systolic and diastolic $\left[\mathrm{Ca}^{2+}\right]_{j}$ and $\left[\mathrm{Ca}^{2+}\right]_{\mathrm{sl}}$, and how impaired TBX5 disrupts calcium handling in atrial myocytes. In addition, a subsequent study needs to be conducted on PITX2 as the next step towards a more comprehensive understanding of the mechanisms by which impairments in the TBX5dependent regulatory network can lead to AF.

\section{Conclusion}

Remodeling of $\mathrm{I}_{\mathrm{K} 1}$ and $\mathrm{I}_{\mathrm{CaL}}$ due to impaired TBX5 are the main contributors to the generation of EAD and
STAP/DAD due to the elevation of systolic and diastolic $\left[\mathrm{Ca}^{2+}\right]_{\mathrm{j}}$ and $\left[\mathrm{Ca}^{2+}\right]_{\mathrm{sl}}$, which activates both $\mathrm{I}_{\mathrm{CaL}}$ and $\mathrm{I}_{\mathrm{NaCa}}$ in triggering EADs and STAPs/DADs. Sufficient inhibition of either $\mathrm{I}_{\mathrm{CaL}}$ or $\mathrm{I}_{\mathrm{NaCa}}$ can suppress both EADs and STAPs/DADs, and hence are possible AAD targets for AF patients with impaired expression of TBX5.

\section{Acknowledgements}

This study was funded by the Health Research Council of New Zealand.

\section{References}

1. Benjamin, E.J., et al., Prevention of atrial fibrillation: report from a national heart, lung, and blood institute workshop. Circulation, 2009. 119(4): p. 606-618.

2. Waks, J.W. and P. Zimetbaum, Antiarrhythmic drug therapy for rhythm control in atrial fibrillation. Journal of cardiovascular pharmacology and therapeutics,2017. 22(1): p3-19.

3. Lubitz, S.A., et al., Association between familial atrial fibrillation and risk of new-onset atrial fibrillation. Jama, 2010. 304(20): p. 2263-2269.

4. Zhou, M., Y. Liao, and X. Tu, The role of transcription factors in atrial fibrillation. Journal of thoracic disease,2015. 7(2): p. 152. 5. Nadadur, R.D., et al., Pitx 2 modulates a Tbx5-dependent gene regulatory network to maintain atrial rhythm. Science translational medicine, 2016. 8(354): p. 354ra115-354ra115.

6.Iwasaki, YK,et al, Atrial fibrillation pathophysiology: implications for management.Circulation,2011.124 :p2264-74.

7. Grandi, E., et al., Human atrial action potential and $\mathrm{Ca}^{2+}$ model: sinus rhythm and chronic atrial fibrillation. Circulation research, 2011: p. CIRCRESAHA. 111.253955.

8. Courtemanche, M, R.J Ramirez, and S Nattel, Ionic mechanisms underlying human atrial action potential properties: insights from a mathematical model.American Journal of Physiology-Heart \& Circulatory Physiology,1998.275: p H301.

9. Bai, J., et al., Ionic and cellular mechanisms underlying TBX5/PITX2 insufficiency-induced atrial fibrillation: Insights from mathematical models of human atrial cells. Scientific reports, 2018. 8(1): p. 15642.

10. Nattel, S., B. Burstein, and D. Dobrev, Atrial remodeling and atrial fibrillation: mechanisms and implications. Circulation: Arrhythmia and Electrophysiology, 2008. 1(1): p. 62-73.

11. Volders, P.G., et al., Progress in the understanding of cardiac early afterdepolarizations and torsades de pointes: time to revise current concepts. Cardiovascular research, 2000. 46(3): p. 376.

12. Weiss, J.N., et al., Early afterdepolarizations and cardiac arrhythmias. Heart rhythm, 2010. 7(12): p. 1891-1899.

13. Choi, B.R., F. Burton, and G. Salama, Cytosolic $\mathrm{Ca}^{2+}$ triggers early afterdepolarizations and Torsade de Pointes in rabbit hearts with type 2 long QT syndrome. The Journal of physiology, 2002. 543(2): p. 615-631.

Address for correspondence:

Dr. Jichao Zhao

Auckland Bioengineering Institute, University of Auckland, New Zealand,

E-mail: j.zhao@ auckland.ac.nz 